\title{
A Qualitative Study on the Inner Feelings and Needs of Medical Students during the Closure Period
}

\author{
Yun-Jie Tong ${ }^{1}$, Yan Guo ${ }^{1}$, Mei-Fen Lv ${ }^{3}$, Yan-Mei Liu ${ }^{2, *}$, Shi-Yun Tan ${ }^{1}$ \\ ${ }^{1}$ Shaanxi University of Chinese Medicine, Xianyang, China. \\ ${ }^{2}$ Shaanxi Traditional Chinese Medicine Hospital, Xi'an, Shaanxi, China. \\ ${ }^{3}$ Qujing First People’s Hospital, Qujing, Yunnan, China.
}

How to cite this paper: Yun-Jie Tong, Yan Guo, Mei-Fen Lv, Yan-Mei Liu, Shi-Yun Tan. (2020) A Qualitative Study on the Inner Feelings and Needs of Medical Students during the Closure Period. International Journal of Clinical and Experimental Medicine Research, 4(4), 266-271.

DOI: 10.26855/ijcemr.2020.10.024

Received: October 20, 2020

Accepted: November 24, 2020

Published: December 2, 2020

*Corresponding author: Yan-Mei Liu, Shaanxi Traditional Chinese Medicine Hospital, Xi'an, Shaanxi, China.

Email: 1305999760@qq.com

\begin{abstract}
To understand the inner feelings and needs of medical students during the closure of the school, so as to provide evidence for strengthening the mental health education of medical students during the closure of the school. Methods: from May to June, 2020, 12 medical students were selected for face-to-face and semi-structured interviews by objective sampling method. Colaizzi 7-step analysis method was used to analyze the interview data. Results: During the closure of COVID-19, medical students' psychological feelings and needs were divided into five themes: psychological feelings: anxiety; Fear; needs: learning needs; the demands of daily life; the need for social support. Conclusion: COVID-19 is undoubtedly a special challenge to China. In the interview with the medical students, some bad emotions such as fear and anxiety appeared. However, most of the respondents expressed the hope that the epidemic would pass quickly and return to a normal life. They mentioned that as a medical student, they felt responsible and would study hard to become an excellent medical worker. Our medical students showed the spirit of hard struggle and independence, which laid a good ideological foundation for their later medical work. However, due to the implementation of COVID-19 campus closed management, some problems are worthy of attention and consideration of the management departments of colleges.
\end{abstract}

\section{Keywords}

COVID-19, Medical Students, Inner Feelings and Needs, Qualitative Research

\section{Introduction}

In December 2019, COVID-19 [referred to as COVID-19, WHO officially named COVID-19 disease 2019 (COVID-19) on February 11, 2020] emerged and gradually spread in Wuhan, by January 30, 2020, there have been nearly 10,000 confirmed cases in China [1]. More than the number of infections from severe acute respiratory syndrome (SARS) in 2003, and the number of cases is rising. In the face of this public health emergency, due to its uncertainty and complexity, the public is prone to psychological stress reactions, such as hypochondriasis, anxiety, depression, obsessive psychology, etc. which, if not timely intervened, may cause a series of problems such as weakened immunity and negative emotions [2]. COVID-19 is mainly transmitted through respiratory droplets and close contact, and confirmed cases have been reported in all provinces in China, with Wuhan in Hubei province 
having the most confirmed cases. In order to prevent its spread, various regions and departments in China have taken effective measures to ensure the safety of people's lives. Shaanxi Provincial Department of Education issued a notice, from April 27, students in Colleges and universities can start to return to school at different times [3]. To this end, from April 27, the students of all majors in our school returned to school orderly in stages and batches. After returning to school, the school adopted closed campus management to ensure the life safety of teachers and students. In order to understand the inner feelings and needs of medical students under the dual pressure of the COVID-19 and the closure of the school, we used the qualitative research method and conducted in-depth interviews with medical students in our school as the main body, in order to provide a basis for strengthening the mental health education of medical students during the closure period of the COVID-19.

\section{Objects and methods}

\subsection{Object of Study}

In this study, objective sampling was used to conduct qualitative interviews with 12 medical students under closed management from May to June in 2020 in our school.

Inclusion criteria: (1) full-time medical students enrolled uniformly by the state; (2) continuous school time of 15 days or more; (3) smooth language expression and clear thinking; (4) to understand the purpose of this research and participate in this interview voluntarily. Exclusion criteria: students who are absent for more than 12 hours due to physical and/or psychological problems during school closure.

The sample size is based on the principle that no new topics appear and data repetition reaches saturation. A total of 12 medical students from the school of Pharmacy, School of Nursing and School of Public Health were selected as the research objects; 3 students from the School of Pharmacy, 6 students from the School of Nursing and 3 students from the School of Public Health; Among them, there are 3 boys and 9 girls; Since only grade 19 graduate students in our school are in closed management this term, all the research objects are grade 19. General information of the research objects is shown in Table 1.

Table 1. General information of the research subjects

\begin{tabular}{cccccc}
\hline serial number & grade & gender & age & major & To get to school time \\
\hline N1 & Grade 19 Graduate student & male & 25 & School of pharmacy & On May 9th \\
N2 & Grade 19 Graduate student & male & 27 & School of pharmacy & On May 10 \\
N3 & Grade 19 Graduate student & female & 25 & School of pharmacy & On May 10 \\
N4 & Grade 19 Graduate student & female & 23 & School of Nursing & On May 10 \\
N5 & Grade 19 Graduate student & female & 24 & School of Nursing & On May 9th \\
N6 & Grade 19 Graduate student & female & 24 & School of Nursing & On May 9th \\
N7 & Grade 19 Graduate student & female & 30 & School of Nursing & On May 9th \\
N8 & Grade 19 Graduate student & female & 32 & School of Nursing & On May 10 \\
N9 & Grade 19 Graduate student & female & 26 & School of Nursing & On May 10 \\
N10 & Grade 19 Graduate student & male & 25 & school of public health & On May 10 \\
N11 & Grade 19 Graduate student & female & 24 & school of public health & On May 9th \\
N12 & Grade 19 Graduate student & female & 24 & school of public health & On May 10
\end{tabular}

\subsection{Research Method}

In this study, descriptive phenomenological research methods in qualitative research were adopted. The researchers conducted face-to-face and semi-structured in-depth interviews with the subjects.

According to the purpose of this research, the interview outline was determined by referring to relevant literature and consulting expert opinions: (1) Could you talk about the daily arrangement during the closed school management period? (2) Is there any difference between your real school closure life and your expected school closure life? Can you talk about it in detail? (3) What is your deepest feeling during the COVID-19 closure? Does it create stress? (4) In this process, what kind of support and help do you hope to get? According to the actual situation during the 
interview, the order of questions can be adjusted appropriately. The privacy of interviewees and humanistic care should be paid attention to in the whole process.

\subsection{Methods of data collection}

Before the interview, the researcher explained the purpose and form of the study to the subjects, and informed them that the whole interview process should be recorded. After the consent of the subjects was obtained, the two sides jointly determined the interview locations, most of which were dormitories and classrooms. The research time was selected when the students had been in the school for more than 15 days with fully closed management. The interview time of each student was controlled within 25 to 35 minutes. During the interview, communication skills can be appropriately applied to encourage interviewees to express their innermost feelings. At the same time, it is necessary to closely observe the changes of non-verbal expressions such as the body, expression and emotion of the research object, and record them in writing if necessary, so as to better understand their ideas.

\subsection{Methods of data analysis}

At the end of the interview, the researcher converted the audio recordings combined with the subjects' performance during the interview into written records within 24 hours. At the same time, each respondent was sequenced in turn according to N1 N12 and their own files were established. The data were analyzed by colaizzi7-step analysis method: read the whole interview record repeatedly, condensing out the meaningful ideas, encoding and summarizing the repeated ideas in the interview, describing them carefully, extracting the theme concepts, and sending them back to the interviewees to confirm whether they are real ideas. To fully understand the situation of medical students during the closure of the school.

\section{Results}

Five themes were extracted from the psychological feelings and needs of medical students during the closure of COVID-19.

\subsection{Psychological Feelings}

Theme 1: Fear. With the extension of school closure time, the subjects consistently expressed that they would feel unsafe even wearing masks in the context of eating, offline learning and other external environments. (1) Fear in daily life. N4: "I went to class one day and the girl next to me coughed once or twice during a class, which scared me so much that I repeatedly pressed the wire on the bridge of my nose of my mask. (Pressing his hand on the mask)." N7: "When I go to buy food in the canteen, I always try to go to the window with fewer people. The window with tinfoil and rice I want to eat is crowded. I have never eaten it before when I come to school. (Shaking his head in frustration)." N11: "Experts say I need to exercise during this period. I run on the playground, breathless with a mask and afraid of being infected without one." (2) The fear of examination. N2: "What I was most afraid of when I came to school was the final exam of medical statistics. I'm weak in math, but this year I taught online, so I was really uncertain and afraid of failing." N5: "Before we came to school, the teacher said epidemiology asked us to write a summary report, but now we have to produce a set of papers. (Sigh)."

Theme 2: Anxiety. The reasons for the anxiety of students from different regions and genders in the interview are different.N3 (Shaanxi): "I want to go to school when I am at home, but When I come to school, I can't go out. Before, I made an appointment with my friend to cook dinner at the place where she lives. Now I can't go out either.” N6 (Sichuan): "I don't think it makes much difference to me when I come to school to proofread. I don't have a place to go anyway. I just can't get used to school meals and don't know what to eat everyday." N2 (male): "I don't know when I'm going to get out, but if I keep going longer, my girlfriend and I won't just fight. (Laughter)." N10 (female): "My tutor contacted me the other day to ask how the paper was going and whether the first draft was finished yet. I really do not know how to do, looking at the students around a lot have already had the results, I also anxious ah! (Sigh) It'll have to be one step at a time.”

\subsection{Requirements}

Theme 3: Learning requirements. During the COVID-19 closure period, the normal study and life were disrupted, the hospital probation activities, operational skills and experimental courses were suspended to online learning. N1: "We need to do experiments for the course we study, but now the situation is not allowed, the school let us do experiments on a program, we use the mouse to do specific operations, but after the experiment, after a 
day or two, forget, feeling better in the lab.” N9: "Before, we had weekly group meetings and had a lot of opportunities to do academic networking, but these days it's not like last year, when all the networking was online and I still have a web browser, so I feel like I missed a lot of learning opportunities.”

Theme 4: Requirements of daily life. Closed management makes students' life inconvenient and their spiritual life unfulfilled. N3: "I've put on a lot of weight at home over the past few months. I was going to work out when I came to school, but the gym wasn't open." N8: "Now there's only one school that sells fruit, so you can only buy it there. It feels like a luxury to eat it once." N12: "because only part of the students began school, the canteen window is also open less, now open these several are tired of eating."

Theme 5: Need for social support. Due to closed management, students' communication with the outside world is limited. The interview found that the vast majority of students are eager to get care and help. N4: "these two days is the most busy time at home orchard, want to chat with my mother, but afraid to disturb her, miss her ah, if I can go home, can also help her ease.” N13: "Almost all of my former undergraduate friends went to work. They had no time to talk to me. I felt so lonely." N11: "I'm having my teeth corrected now. I need to go to the stomatological hospital regularly. Every time it’s time, I have to ask the teacher for leave. (Laughter).”

\section{Discuss}

From the phenomenological perspective, this study describes the psychological feelings and needs of medical students during the closure of COVID-19, and analyzes the reasons mainly related to students themselves, campus management and social support.

\subsection{Students themselves}

The students in the closed school were unable to go out for a long time and worried about being infected, which had a huge impact on their psychology. This is consistent with the research results of Lu Juan et al. [5] during the closure of the school by SARS in 2004. In the interview, it was found that almost every student had a negative emotional reaction. They often felt fear and anxiety about their daily life and the exams they would have to face in the future. It is also found in the interview that different genders and regions also have an impact on the psychological state of students. Female students and those who are not native place are more dissatisfied with the quality of life on campus than other groups. As a medical student, it is the hope of the future medical career in our country, so we should improve his ability of self-regulation. Studies have pointed out that those with better self-regulation ability seldom have bad living habits, and they usually face life with an optimistic and positive attitude [6]. Similarly, in the study of Pan Guichun et al. [7], the anxiety and depression levels of the experimental group members receiving the self-regulation theory guidance basically returned to normal. It can be seen that effective self-regulation can improve people's negative psychology. Therefore, students should attach importance to cultivation of self-regulation, by distracting, relax, catharsis, talk, and encourage myself to adjust themselves, a comprehensive understanding of its physical and mental conditions, correctly deal with difficulties and setbacks, Gradually improve the relationship between themselves and the outside world, to ensure good control of their own behavior, according to different circumstances, with their own unique adjustment of the correct handling of negative emotions.

\subsection{Campus Management}

During the interview, the interviewees repeatedly mentioned the problem of school meals. This may be related to the reduction of canteen Windows caused by only a small number of students in school at present. According to the actual situation, the administrators should make flexible management, provide diversified, multi-flavor and multi-form food choices to the students who are closed during the "New Championship" period, do a good job in the handover work of each window of the canteen, and quickly solve the concentration problems that students urgently need to solve [8].

The problem of learning style was also a concern of many respondents. At present, our school mainly adopts the online and offline teaching mode. Studies [7] show that the vast majority of students tend to combine offline teaching with online and offline teaching, which is consistent with the results of this study. The mixed online and offline teaching mode has been widely applied in the current course teaching. Compared with the traditional teaching mode, it not only conforms to the development trend of modern education, but also meets the needs of students [10]. Therefore, in the later stage of teaching, the school should pay attention to online teaching on the one hand, considering the objective factors affecting online teaching, and on the other hand, considering the students' acceptance and understanding of knowledge, and combining with the offline medical practice courses, improve the course organization and deepen the students' understanding of knowledge. In addition, in this special period, school admin- 
istrators should also pay attention to the psychological state of students, through listening, communication and other ways to fully evaluate the problems of the student group, give personalized psychological care, and provide them with corresponding support and help. Online mindfulness training and other relevant courses can be conducted to improve students' anxiety and enhance their learning ability. Mindfulness training is simple, convenient and easy to operate. It can be practiced in groups and has been proven to improve negative psychological problems such as anxiety, depression and compulsion [11-12]. Scholars Such as Yang Qun et al. [13] used this method to intervene medical undergraduates in military schools, and the research results showed that mindfulness training could effectively reduce the anxiety level of students and significantly improve their self-control ability and learning ability.

\subsection{Social support}

In this study, it is found that when students encounter psychological problems, they seldom turn to the psychological counseling room, and most of them will choose to talk to their friends and relatives to solve their psychological problems. Social support refers to mutual support and help among people, especially in the face of difficulties or setbacks, support becomes more important [14]. Studies have shown that when individuals have a better social support system, they will have richer material and spiritual resources, and the higher the level of social support, the lower the level of psychological distress [15-18], which will encourage them to face difficulties with a positive attitude. Therefore, as a school administrator, we should understand the living conditions of students, further strengthen the social support for students, and play an active role in the protection of students' life and health. Heroes can be invited to share their frontline stories, so that students can deeply feel the responsibility and responsibility of a medical student, understand the hardships and difficulties of controlling the epidemic, and understand the school closure regulations. As family members, they should pay close attention to their children's emotional changes, provide comfort and inspiration, encourage students to adopt a positive attitude towards life, resume necessary social activities, and take part in more extracurricular activities to meet the needs of self-realization. In addition, students can communicate more with their friends to get support and help.

\section{Summary}

The COVID-19 epidemic in 2020 will undoubtedly pose a special challenge to China. In the interview with the medical students, some bad emotions such as fear and anxiety appeared. However, in the face of the real closed school life, they can abide by the rules and regulations of the school, do their own protection, and cooperate with the management work related to the epidemic. Report your health condition on time every day, and try to reduce unnecessary leave. Our medical students showed the spirit of hard struggle, independence and self-improvement, which laid a good ideological foundation for their later medical work. Of course, due to the implementation of the COVID-19 campus closed management, this is undoubtedly a severe test for the education management departments at all levels in the school. Some of these problems should be paid attention to and considered by the administrative departments of colleges.

\section{Acknowledgements}

This study was approved by 2019 Higher Education Scientific Research Project of Shaanxi Institute of Higher Education (XGH19152) and the Innovation project for graduate students of university-level scientific research projects of Shaanxi University of Traditional Chinese Medicine in 2020 for funding the project.

\section{References}

[1] Chinese Center for Disease Control and Prevention, National Health Commission. Novel Coronavirus pneumonia Outbreak Update at 24:00, 30 January [EB/ OL] 2020-01-31 [2020-04-18].

[2] Wang Yiniu, Luo Yuejia. (2003). Features and responses of mood disorders in public health emergencies [J]. Advances in psychological science, 2003, 11 (4): 387-392.

[3] Ministry of Education, PRC. Shaanxi college students on April 27 peak back-to-school. http://www.moe.gov.cn/jyb _xwfb/xw_zt/moe_357/jyzt_2020n/2020_zt03/zydt/zydt_dfdt/cjkxzb/202004/t20200421_445176.html, 2020-04-21.

[4] Tan Yan, Li Xuying, Huang Gang, Wei Tao, Peng Siyi, Li Xingfeng, Lin Qin. (2020). Study on the desirability of nurses’ rotation in oncology hospital [J]. Chinese Journal of Clinical Nursing, 2020, 12(1): 56-58.

[5] Jin Ningning, Zuo Yue-ran, Zhang Xin, Tang Hong-yuan, Ma Xuan. (2004). Influence of closed management on nursing students' Psychology during SARS [J]. Nursing research, 2004, 18(3): 226-227.

[6] Cai Dongdi, Liang Lifen, Pan Guichun, Wang Helei, Pang Junchen. (2012). Effects of self-regulation theory on anxiety and 
depression status and quality of life of patients [J]. Electronic Journal of Modern Medicine and Health Research, 2012, 2(16): 130-131.

[7] Pan Guichun, He Rubing, Cai Dongdi, Liang Li-fen, Wang He-lei, Pang Jun-chen. (2017). Application of self-regulation theory in patients with anxiety and depression [J]. Nursing practice and research, 2017, 14(10): 140-142.

[8] Zhang Yimei. (2020). Exploration of strategies for improving catering Management in Colleges and Universities [J]. University Logistics Research, 2020(01): 25-27.

[9] Meng Aiqing, Li Xiaoli, Wu Shanshan. (2020). An investigation and Analysis of college students’ online learning During the COVID-19 epidemic [J]. Journal of Dezhou College, 2020, 36(02): 1-4+12.

[10] Li Jing. (2019). Qualitative research on the effect of Mixed teaching on online learning of medical students [J]. Science and Technology Vision, 2019, 30: 161-162.

[11] Gao Qian, Song Jia, Zou Yuan. (2017). The effect of focused solution model combined with mindfulness intervention on cancer-induced fatigue and sleep quality in patients with gastric cancer [J]. Qilu Med J, 2017, 32(4): 483-486.

[12] Xiong Weirui, Yu Lu. (2011). Mindfulness therapy—a new psychotherapy approach [J]. Medicine \& Society, 2011, 24(1): 89-91.

[13] Yang Qun, Chi Yuhao, Lu Kai, Wang Bingzhao, Wu Lin, Wang Yidi, Cui Di, Dai Hong, Pan Ying, Hu Xinyu, Xiao Wei. (2008). Study on the Improvement of Anxiety, self-control and Learning Effect of mindfulness Training for medical undergraduates in military Schools [J]. Air Force Medical Journal, 2008, 34(04): 279-280.

[14] Jane Norbeck, Zhou Yutong. (1990). What is Social support [J]. Chinese Journal of Practical Nursing, 1990, 01: 44+50.

[15] Zhang Zhuo. (n.d.). The relationship between positive coping style, personality traits and perceived social support of post-00s college students [J]. Chinese Journal of Health Psychology 1-8.

[16] Gao Yongli, Lin Tao, Cao Yu, Li Xuemei. (2020). Relationship between psychological distress, social support and psychological resilience of male nurses in Sichuan province under the COVID-19 epidemic [J]. Journal of Chengdu Medical College, 2020, 02: 178-181+188.

[17] Wang Li. (2012). Research on the relationship between social support and mental health of junior high school students [J]. Chinese Journal of Health Psychology, 2012, 20(1): 91-92.

[18] Shi JY, Chen ZT, Yin F, et al. (2016). Resilience as moderator of the relationship between left-behind experience and mental health of Chinese adolescents [J]. Int J Soc Psychiatry, 2016, 62(4): 386-393. 